\title{
Escala de modos de afrontamiento: consideraciones teóricas y metodológicas"
}

\author{
Scale of Ways of Coping: Theoretical and
} Methodological Considerations

CARLOS NAVA-QUIROZ
Recibi
CyNTHIA ZAIRA VEGA-VALERO
Rocío SORIA TRUJANO
Facultad de Estudios Superiores Iztacala, UNAM, México

Artículo de investigación en psicometría.

** Av. De los Barrios no. 1, los Reyes Iztacala, Tlalnepantla, Edo. De México, C.P. 54090, Correos electrónicos: canaqi@servidor.unam.mx; czaira. vega@correo.unam.mx; rociyito@yahoo.com.mx

\begin{abstract}
RESUMEN
El objetivo fue reconsiderar las propiedades métricas de la escala de modos de afrontamiento, a partir de: la comparación de los factores originales y los obtenidos en este estudio; evolución de la estructura factorial para nuestros datos y comparación con la original; valoración de la fiabilidad de distintas estructuras factoriales; y, estimación de los niveles de predicción del afrontamiento y apoyo social respecto del estrés. La muestra fue de 250 participantes voluntarios. Los análisis factoriales, de fiabilidad y de regresión mostraron que no hay evidencia para considerar que la escala sea multidimensional y sí unidimensional. No existe sustento teórico que permita suponer la validez de conceptos teóricos o factores subyacentes en dicha escala, y los análisis empíricos así lo muestran. El nivel real de la escala, es de carácter general. Palabras clave autores

Modos de afrontamiento, confiabilidad, apoyo social, estrés.

Palabras clave descriptor

Análisis factorial, actitud, psicología, estrés psicológico.

A B S T R A C T

The objective was to reconsider the metric properties of the scale of ways of coping from: the comparison of the original factors and the obtained ones by us; to evaluate the factorial structure our data and to compare it with the original one; to value the reliability of different factorial structures and; to consider the levels of prediction of the coping and social support respect to stress. The sample was 250 voluntary participants. The factorial analyses, of reliability and of regression showed that there is evidence no to consider that the scale is multidimensional and if one-dimensional. Theoretical sustenance does not exist that allows to suppose the validity of theoretical concepts or underlying factors in this scale and the empirical analyses therefore show it. The real level of the scale is of general character.

Key words authors

Ways of Coping, Reliability, Social Support, Stress.

Key words plus

Factor Analysis, Attitude, Psychology, Stress Psychology.
\end{abstract}


Estrés y afrontamiento están vinculados de manera directa. El modo en que los individuos enfrentan las situaciones tiene que ver con los niveles de estrés, además de otros factores, como la percepción de apoyo social. Por esta razón, las estrategias para medir el estrés y el afrontamiento son trascendentes. En particular la escala de modos de afrontamiento, que ha servido de base para desarrollar otras, es controvertida por sus propiedades métricas. Es el propósito de este trabajo hacer una reconsideración crítica para evaluar de nuevo la escala de afrontamiento propuesta por Lazaruz y Folkman, (1991), para determinar si es o no una escala multidimencional, así como sus niveles de fiabilidad y validez.

En el modelo teórico de estrés-afrontamiento de Lazarus y Folkman (Folkman, Lazarus, DunkelSchetter, DeLongis \& Gruen, 1986; Folkman, Lazarus, Gruen \& DeLongis, 1986; Lazarus \& Folkman, 1991) existen dos procesos: evaluación cognitiva y afrontamiento, como mediadores de la relación estrés, individuo-ambiente y sus resultados a corto y largo plazo. La evaluación cognitiva es un proceso a través del cual el individuo valora si algún evento ambiental al cual se enfrenta es relevante o amenazante para su bienestar y de qué manera. A la vez, se divide en evaluación primaria y secundaria, cuya distinción radica en el tipo de preguntas que el individuo se plantea. Para la primera, se pregunta si el evento en cuestión es benéfico o perjudicial para su salud o bienestar, y, en la segunda, si puede hacer algo por alterar o cambiar la situación.

En lo referente al afrontamiento, Lazarus y Folkman (1991, p.164) lo definen como "... aquellos esfuerzos cognitivos y conductuales constantemente cambiantes que se desarrollan para manejar las demandas específicas externas y/o internas que son evaluadas como excedentes o desbordantes de los recursos del individuo". Por lo que el afrontamiento es considerado como un proceso dinámico, que involucra la evaluación y reevaluación constante de las personas ante situaciones demandantes. Así, la función del afrontamiento está en consonancia con las estrategias que los individuos llevan a cabo, para la consecución de objetivos particulares.
Las estrategias, propuestas por Lazarus y Folkman (1991) son conocidas como el afrontamiento dirigido a la acción, que son actividades o manipulaciones orientadas a modificar o alterar el problema, y el afrontamiento dirigido a la emoción, que son las acciones que ayudan a regular las respuestas emocionales a las que el problema da lugar, "En general, las formas de afrontamiento dirigidas a la emoción tienen más probabilidad de aparecer cuando ha habido una evaluación de que no se puede hacer nada para modificar las condiciones lesivas, amenazantes o desafiantes del entorno. Por otro lado, las formas de afrontamiento dirigidas al problema son más susceptibles de aparecer cuando tales condiciones resultan evaluadas como susceptibles de cambio..." (Lazarus \& Folkman, 1991, p. 172).

En particular, Lazarus y Folkman (1991, p. 336) afirman que los instrumentos que miden el afrontamiento, de manera general, son inespecíficos para saber qué se está afrontando y aseveran que "En el curso de nuestras propias deliberaciones nos hemos ido convenciendo cada vez más de que tenemos que alejarnos de evaluaciones globales y dirigirnos a otras específicas, de modo que podamos determinar qué es lo que se está afrontando".

Con este sustento teórico los citados autores se dan a la tarea de construir un instrumento que mida el afrontamiento, así la escala de modos de afrontamiento es elaborada a partir de entrevistas que permiten especificar reactivos, los que son puestos a prueba en diferentes momentos (DunkelSchetter, Folkman \& Lazarus, 1987; Folkman \& Lazarus, 1980; Folkman \& Lazarus, 1985; Folkman \& Lazarus, 1988; Folkman, Lazarus, DunkelSchetter, Delongis \& Gruen, 1986). Los mismos escritores manifiestan que "La investigación futura pondrá en claro si existe o no un conjunto invariable y relativamente estable de factores que describen la estructura del afrontamiento, tal como se mide de acuerdo con este planteamiento." (Lazarus \& Folkaman, 1991, p. 337).

El análisis de la literatura, tanto de habla inglesa como castellana, muestra inconsistencias: baste con revisar las propias investigaciones de Folkman y Lazarus (Dunkel-Schetter, Folkman \& Lazarus, 
1987; Folkman \& Lazarus, 1980; Folkman \& Lazarus, 1985; Folkman \& Lazarus, 1988; Folkman, Lazarus, Dunkel-Schetter, Delongis \& Gruen, 1986) para percatarse de que la estructura factorial en los análisis cambia, así como la cantidad de ítems y en ocasiones su contenido. Investigadores, independientes al proyecto de Lazarus y Folkman, reportan estructuras factoriales diversas, que van de dos a ocho factores con diferentes niveles de saturación, fiabilidad y cantidad de ítems por factor, entre otras (Aldwin \& Revenson, 1987; Atkinson \& Violato, 1994; Parker, Endler \& Bagby, 1993; Parkes, 1984, 1986; Scheier, Weintraub \& Carver, 1986; Vitalino, Russo, Carr, Majuro \& Becker, 1985).

Análisis de traducciones castellanas revelan resultados similares a los antes citados, (para mostrar lo anterior elegimos dos artículos, en especial por su sistematicidad) por ejemplo, Sotelo y Maupome (1999) traducen, evalúan y estandarizan la escala de modos de afrontamiento de Lazarus y Folkman (1985), de 66 ítems, y reportan que no fue posible reproducir los ocho factores iniciales, encontraron sólo seis, es decir una estructura factorial nueva, el alfa de Cronbach general 0.86, para la escala es elevada y oscila de 0.57 a 0.90 para los factores. En otro estudio, Ibáñez y Olmedo (2003) retoman la escala de modos de afrontamiento (Lazarus \& Folkman, 1991), y encuentran seis factores los que comparan con la estructura original encontrada por Lazarus y Folkman y no notan semejanza de las mismas, los alfas de Cronbach para los seis factores oscila de 0.01 a 0.68 y no reportan un índice general, incluso desaconsejan el uso de la escala.

En la comparación de ambos estudios, la versión de la escala de modos de afrontamiento es la misma y obtienen resultados similares, sólo seis factores e índices de fiabilidad más o menos aceptables para los mismos, pero al comparar la composición de los factores, de un estudio a otro, no existe semejanza en el contenido de los ítems que componen cada factor. Por ejemplo, el primer factor de Sotelo y Maupome (1999), está compuesto por 15 ítems del 6 a 20, mientras que para Ibáñez y Olmedo (2003) el primer factor sólo tiene 10 ítems $(34,46,26,39,20,23,30,36,38,56)$ y comparten el ítem 20 en este factor, lo mismo ocurre cuando comparamos el total de los factores.

Otros autores critican la validez de contenido de la escala, con base en el análisis del marco teórico y la pertinencia en la construcción de los ítems (Aliaga \& Capafóns, 1996), lo que denota que en la teoría del estrés-afrontamiento no existen constructos como afrontamiento confrontativo, autocontrol, búsqueda de apoyo social, entre otros.

Dado lo anterior, una conclusión es que ante la inconsistencia de los factores, de un estudio a otro, se trata de una escala que no es multidimensional. De hecho, el marco teórico bajo el que está construida no permite, en principio, hablar de modos específicos de afrontamiento - más allá del afrontamiento dirigido al problema o la emoción-y una de las esperanzas de los autores (Folkman \& Lazarus, 1991) era que la investigación empírica les ayudara a proponer dichos conceptos teóricos.

En el modelo de estrés-afrontamiento, este último regula los niveles de estrés, lo mismo ocurre con el apoyo social (Cohen, Gottlieb \& Underwood, 2000). Existe evidencia suficiente que demuestra los efectos benéficos del apoyo social percibido en la regulación del estrés y la salud (Lakey \& Cohen, 2000; Wills \& Shinar, 2000). La perspectiva del estrés-afrontamiento propone que el apoyo social contribuye a la salud protegiendo a la gente de los efectos negativos del estrés (Lakey \& Cohen, 2000). Así, en la tradición del estrés-afrontamiento la percepción del apoyo social enfatiza su disponibilidad y opera como reductor del estrés -lo que conlleva a la mejora de la salud-y es explicado por la hipótesis de regulación: "Un modelo propone que el apoyo está relacionado con el bienestar sólo (o primariamente) para los individuos bajo estrés. Este es denominado como modelo de regulación porque postula que el apoyo 'regula' (protegiendo) a las personas de influencias patológicas potenciales de los eventos estresantes" (Cohen \& Wills, 1985, p. 310). Lo anterior implica que entre el apoyo social y el afrontamiento no existe relación directa, pero sí una relación de dependencia de éstos dos con el estrés.

En concordancia con el análisis previo, no esperamos encontrar la misma estructura factorial que 
en los estudios originales, lo que quiere decir que fracasará la comprobación de que es multidimensional, lo que parece lógico, si recordamos que la escala fue construida a partir de los postulados de afrontamiento dirigido a la acción y a la emoción, esto es, que puede ser bidimensional o unidimensional según lo muestren los análisis métricos. Un argumento adicional está basado en los niveles de confiabilidad encontrados en los estudios antes citados. Hay que recordar que la fiabilidad es un requisito previo a la validez del constructo y entre más baja sea la primera la cantidad de error debida al azar incrementa (Cortada, 1968, 2000).

Por lo antes dicho, el propósito del presente estudio fue reconsiderar las propiedades métricas de la escala de modos de afrontamiento (versión de Lazarus, Folkman, Dunkel-Schetter, DeLongis \& Gruen, 1986) a partir de: a) la comparación de los factores reportados por los autores originales y los obtenidos en este estudio, b) la evaluación de la mejor estructura factorial para nuestros datos y su comparación con la original, c) la valoración de los niveles de fiabilidad de distintas estructuras factoriales, y d) el desarrollo de un análisis multivariado, con el fin de estimar los niveles de predicción del afrontamiento y apoyo social respecto del estrés.

\section{Método}

\section{Participantes}

Fueron 250 voluntarios, $75 \%$ mujeres. Con un promedio de edad de 31.07, y desviación estándar de 17.29. En el nivel educativo, $12.7 \%$ reportaron primaria, $9 \%$ secundaria, $2.5 \%$ bachillerato, $9.6 \%$ carrera técnica y nivel de licenciatura $66 \%$. Reportaron trabajar $34.5 \%$.

\section{Instrumento}

Fue incluido un formato de datos demográficos además de la escala de modos de afrontamiento de Folkman y Lazarus (1986) y Lazarus y Folkman (1991). La escala consta de 66 reactivos, que son afirmaciones respecto de las maneras de afrontamiento, dirigidos a la emoción y al problema. Las opciones de respuesta van de 0 a 3, donde 0 corresponde a "En Absoluto" y 3 a "En gran medida". Los detalles de construcción de la escala están en las obras citadas. La base para la traducción castellana fue el libro Estrés y procesos cognitivos de Lazarus y Folkman (1991) y Lazarus, Folkman, Dunkel-Schetter, DeLongis y Gruen (1986) y fue sometida a verificación por tres psicólogos expertos en el tema y en el idioma inglés. Las modificaciones a la redacción de los reactivos no fueron substanciales.

La versión de escala de modos de afrontamiento que tomamos para comparar, fue la de Folkman, Lazarus, Dunkel-Schetter, DeLongis y Gruen (1986). Las propiedades métricas de fiabilidad ( $\alpha$, alfa de Cronbach) y el análisis factorial (8 factores), con 50 ítems, son: Afrontamiento confrontativo $(\alpha=0.70)$, Distanciamiento $(\alpha=0.61)$, Autocontrol $(\alpha=0.70)$, Búsqueda de apoyo social $(\alpha=0.76)$, Aceptación de responsabilidades $(\alpha=$ 0.66), Evitación-escape ( $\alpha=0.72$ ), Planeación de solución de problemas $(\alpha=0.68)$, Reapreciación positiva $(\alpha=0.79)$.

Inventario de estrés cotidiano (IEC) de Brantley, Waggoneer, Jones y Rappaport (1987) adaptado por Nava, Anguiano y Vega (2003) con alfa de Cronbach estandarizados de 0.9219 y alfas particulares para cada ítem superior a 0.9174.

Escala de Percepción de Apoyo Social (Vaux, Phillips, Holly, Thompson, Williams \& Stewart, 1986) de 23 ítems, con alfa de Cronbach de 0.9018, para este estudio.

\section{Procedimiento}

Las escalas fueron aplicadas en el lugar de trabajo de los participantes. En este caso solicitamos citas específicas con el fin de realizar las aplicaciones y no tener que dejar los cuestionarios y recogerlos con posteridad. En el caso de los que no trabajaban, las aplicaciones fueron hechas en una clínica de Seguro Social y en una escuela de nivel preescolar.

En todos los casos se indicó a los voluntarios que los cuestionarios tenían instrucciones, pero que podían preguntar en caso de tener dudas. 
También se garantizó la confidencialidad de la información y su uso restringido a fines estadísticos.

\section{Variables y análisis de resultados}

Estadísticas descriptivas, para los demográficos.

Alfa de Cronbach, Guttman y Spearman-Brown para evaluar la fiabilidad de las escalas.

Análisis factorial para estimar los factores de afrontamiento.

Análisis de regresión lineal múltiple.

Programa SPSS para procesar la información.

\section{Resultados}

El análisis inicial consistió en intentar reproducir los niveles de fiabilidad y la estructura factorial del estudio original. Para tal fin, se tomaron los ítems que componen cada factor (según, Folkman, Lazarus, Dunkel-Schetter, DeLongis \& Gruen, 1986) para evaluar los niveles de confiabilidad. La Tabla 1 muestra los alfas de Cronbach para el estudio de Folkman et al. (1986) y la réplica del presente estudio (RE); los factores (F) 2, 6 y 7 coinciden con un mínimo de error y los restantes no, en cualquier caso no superaron el 0.80 y el índice de fiabilidad general fue de 0.90 para la réplica.

\section{TABLA 1}

Fiabilidad del estudio original y la réplica con nuestros datos

\begin{tabular}{lllllllll}
\hline \multicolumn{1}{c}{ Estudio } & F1 & F 2 & F 3 & F 4 & F 5 & F 6 & F 7 & F 8 \\
\hline $\begin{array}{l}\text { Folkman et } \\
\text { al. 1986 }\end{array}$ & 0.70 & 0.61 & 0.70 & 0.76 & 0.66 & 0.72 & 0.68 & 0.79 \\
Réplica & 0.54 & 0.62 & 0.57 & 0.65 & 0.60 & 0.72 & 0.66 & 0.67
\end{tabular}

Fuente: elaboración propia.

Tomando en cuenta los propios 50 ítems, no fue posible obtener la estructura factorial original, a pesar de realizar el mismo tipo de factorización y rotación que los originales (factorización principal y rotación oblicua). Debido a lo anterior, la gráfica de sedimentación fue introducida como criterio de decisión, para elegir la cantidad de factores por retener. La gráfica muestra con claridad sólo dos factores (véase Figura 1), el primero muy próximo al autovalor 10 y el segundo al 4, que tienen un $27 \%$ de varianza explicada. Con esta información, solicitamos dos factores al programa.

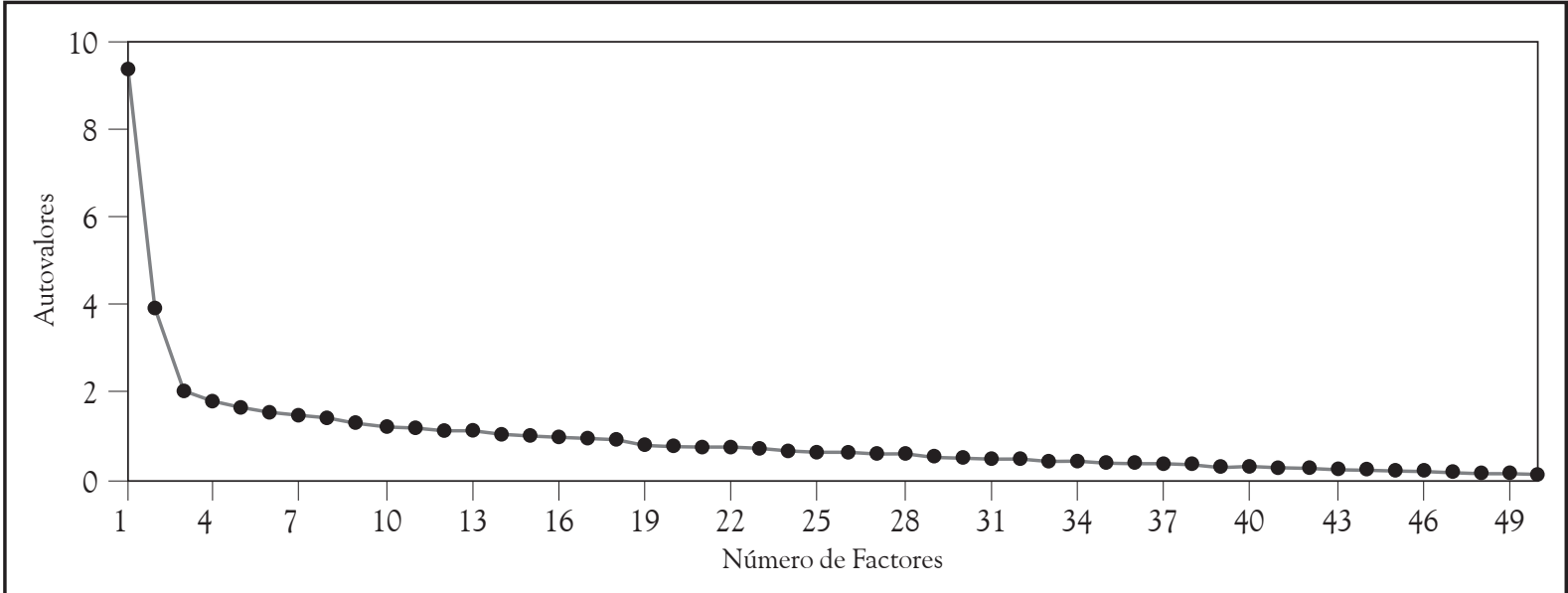

Figura 1

Gráfico de sedimentación.

Fuente: elaboración propia. 
Los análisis de confiabilidad para el primer factor (27 ítems) muestran niveles alfa de Cronbach de 0.8971. En el segundo factor se encuentran 23 ítems con alfa de 0.8094, pero en este caso tres ítems fueron eliminados (1, 60 y 22) por ser negativos con lo que el alfa resultó con 0.8418 de confiabilidad.

Hasta este momento se trabajó con los 50 reactivos del estudio primigenio, por lo que se decidió retomar los 66 ítems, hacer el mismo análisis y compararlo con el de los autores originales.

En este caso, el primer análisis consistió en la evaluación de la fiabilidad de la escala, con tal propósito, los reactivos con correlaciones ítem-total menores de 0.30 fueron eliminados. Así, la escala quedó con 49 ítems y alfa de 0.9232. En comparación con la propuesta de Folkman et al. (1986) resultó un ítem menos y difirieron 10 (5, 24, 27, $32,37,55,57,61,64,65)$ que fueron retenidos y 11 ítems que no aparecieron en nuestro análisis, $(1,7,10,11,14,16,22,33,40,47,60)$ respecto del original.

Con la anterior estructura, fue solicitado el análisis factorial (método de componentes principales y rotación varimax) y la gráfica de sedimentación, la que mostró con claridad dos factores (Figura
2), el primero con autovalores cercanos a 11 y el segundo a 4 , con un total de varianza explicada del 29\%.

En el análisis factorial inmediato solicitamos dos factores; el primero constó de 27 ítems y confiabilidad alfa de 0.8985 , y para el segundo fueron 22 ítems y confiabilidad de 0.8651 .

En la comparación de los factores retenidos por Folkman et al. (1986) y los aquí encontrados, se aprecia que en ambos casos el primer factor es muy parecido, sólo difieren en 3 ítems: 62, 41 y 17 para el estudio de Folkman et al. (1986) y 5, 55 y 27 para el de la presente investigación. Para el segundo factor sólo se coincidió en 12 ítems (6, 9, 12, 13, 15, 21, 29, 43, 44, 58, 50, 59).

En el siguiente análisis de regresión múltiple, la variable por predecir fue el estrés y las que sirvieron para pronosticar fueron la percepción de apoyo social (PAS) y el afrontamiento (AF). En concreto, el AF49 corresponde a este estudio de confiabilidad y tomada la escala como unidimensional, mientras que LF50 son los ítems propuestos por Folkman et al. (1986) y tomados como unidimensionales, al margen de los factores originales. De manera adicional, el análisis también incluyó la propuesta de afrontamiento de Folkman et al. (1986), de dos

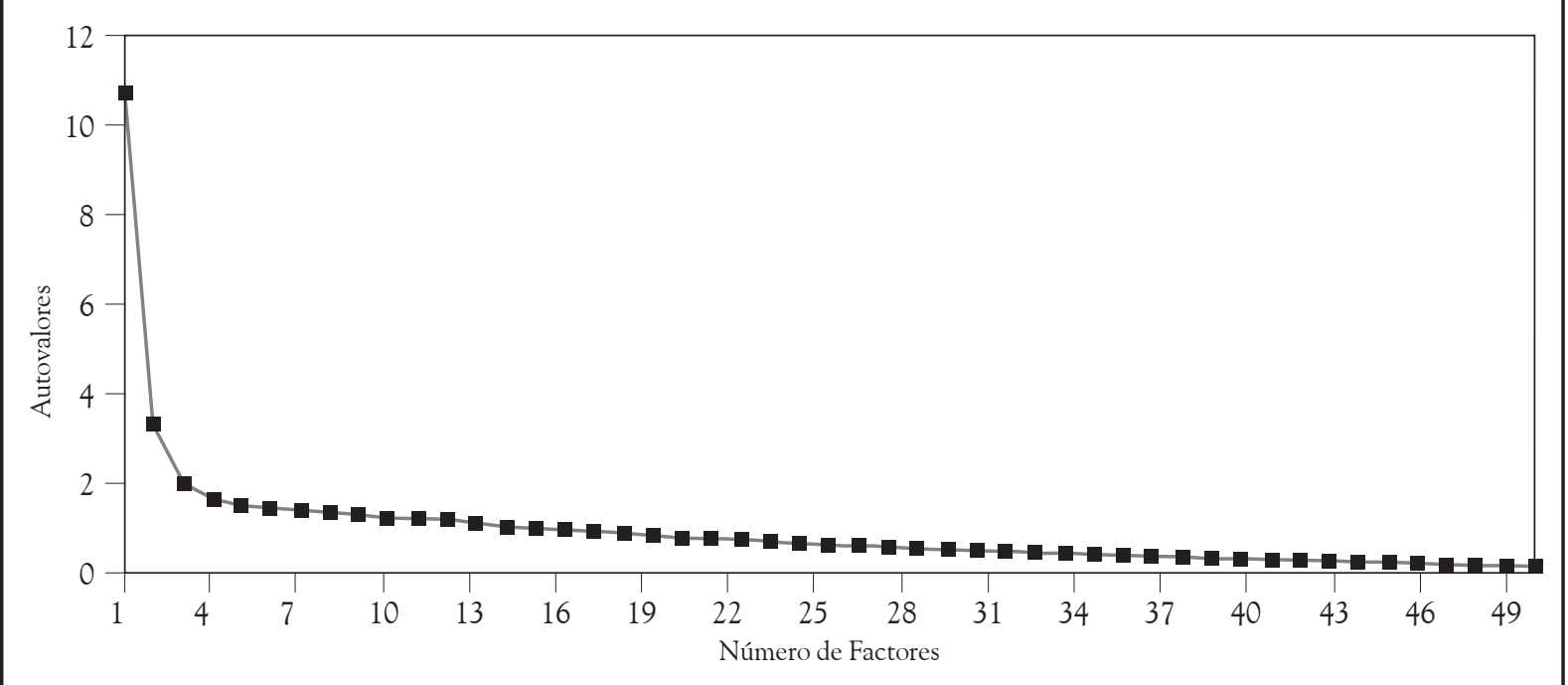

FigURA 2

Gráfico de sedimentación.

Fuente: elaboración propia. 
factores (PAS y dos Factores LF50), lo mismo que la desarrollada aquí (PAS y dos Factores AF49).

TABLA 2

Regresión de apoyo social y diferentes combinaciones de afrontamiento para predecir el estrés

\begin{tabular}{lcccc}
\hline $\begin{array}{c}\text { Variables } \\
\text { predictoras }\end{array}$ & R2 & Error & Sig. de T & Tolerancia \\
\hline PAS y AF49 & 0.118 & 57.29 & PAS .004 & 0.999 \\
& & & AF49.000 & 0.999 \\
PAS y LF50 & 0.120 & 57.21 & PAS .003 & 0.999 \\
& & & 50 LF .000 & 0.999 \\
PAS y 2 Fac- & 0.174 & 55.60 & PAS .015 & 0.974 \\
tores de LF50 & & & F1 .869 & 0.779 \\
& & & PAS .012 & 0.774 \\
PAS y 2 Fac- & 0.166 & 55.68 & F2 .000 & 0.697 \\
tores AF 49 & & & F1 1.386 & 0.634 \\
\hline
\end{tabular}

Fuente: elaboración propia.

En todos los casos (Tabla 2), los coeficientes de determinación son muy semejantes lo mismo que el error. Las pruebas $t$-para las betas del modelo de regresión- fueron significativas para el PAS en todos los casos y cuando la escala se asume como unidimensional. No ocurre lo mismo cuando la escala es tratada como bidimensional, sólo el factor dos es significativo. Por otra parte, cuando los índices de tolerancia son bajos indican que existe colinearidad, lo que quiere decir que las variables con índices bajos son una combinación de las otras. En este caso, la presencia de dos factores la tolerancia baja indica que ambos factores son una combinación, y por lo tanto F1 y F2 son uno sólo y no dos independientes, por lo tanto puede explicarse mejor como unidimensional y no como bidimensional.

Por lo anterior, y bajo el supuesto de que la escala de modos de afrontamiento es unidimensional, se analizó a partir del coeficiente de consistencia interna (división en dos mitades). En este caso, se eliminó el ítem 17 (que fue el que menos correlación ítem-total presentó) con lo que permanecieron 48 y así se tuvo la posibilidad de realizar la comparación por pares (forma I, 24 ítems) y nones (forma II, 24 ítems). Los coeficientes de consistencia interna fueron: de división por mitades de Guttman $=0.8736$ y Spearman - Brown $=0.8766$; para la forma I el alfa quedó de 0.8453 y para la forma 2 de 0.8804. Y el alfa de Cronbach para toda la escala de 0.9228

\section{Discusión}

El objetivo de este trabajo fue reconsiderar las propiedades métricas de la escala de modos de afrontamiento. Los análisis mostraron el mismo tipo de dificultades que las reportadas por otros autores y también indican que no hay argumentos suficientes para pensar que la escala de modos de afrontamiento es multidimensional y sí que es unidimensional.

Desarrollar un estudio que replicara el trabajo de Folkman, Lazarus, Dunkel-Schetter, DeLongis y Gruen (1986), era necesario para corroborar lo que de antemano era conocido: que la escala presenta problemas de validez. La novedad, en este caso, radicó en retomar los ítems reportados por los anteriores autores y someterlos a prueba, tanto en lo que respecta a los niveles de fiabilidad, como en la factorización; en todo caso, las comparaciones no fueron equiparables. En esta circunstancia, una explicación plausible es la manera en que se recogió la información, Folkman et al. (1986) mencionan que sus participantes fueron setenta y cinco parejas (casados), entrevistados en cinco ocasiones diferentes a lo largo de cinco meses (750 observaciones). Desde el punto de vista metodológico implica medidas repetidas, lo que conlleva un sesgo en los niveles de fiabilidad y en la estructura factorial propuesta. Baste con señalar que la estadística tradicional está diseñada para observaciones que son independientes entre sí y la violación de este supuesto puede provocar sesgos importantes.

Pero icómo explicar las inconsistencias entre los estudios que intentan replicar los resultados originales (de Folkman et al., 1986)? Se puede pensar que la respuesta puede estar en el hecho de que la escala no es multidimensional. 
Esto es, los análisis subsecuentes fueron tendientes a construir una nueva estructura factorial, que se inició con la selección de ítems a partir de la fiabilidad de la escala y la revaloración de la propuesta de Folkman et al. (1986), con el fin de probar si la escala podía ser evaluada como unidimensional o multidimensional. El análisis de regresión múltiple fue elegido para poner a prueba la aseveración teórica que declara que el estrés es regulado por el afrontamiento y otras variables como la percepción del apoyo social. Los criterios para evaluar la adecuación del modelo descansan en el coeficiente de determinación, el error, el análisis de varianza, la evaluación de los coeficientes betas individuales y la colinearidad, así como en los niveles de fiabilidad.

En lo concerniente a la fiabilidad Nunnally y Bernstein (1995) afirman que "el contenido de una prueba es homogéneo cuando tiene poco error de medición (un coeficiente $\alpha$ alto) y mide sólo un atributo. La homogeneidad implica que las medidas son matemáticamente unidimensionales (es decir, que los sujetos varían a lo largo de la escala que mide ese rasgo en una sola manera) y unifactoriales..." (p. 349). En general, los $\alpha$ (alfas de Cronbach) para los 8 factores fueron bajos, convenientes cuando fueron tomados como bidimensionales y muy satisfactorios como unidimensionales. A pesar de lo anterior, los $\alpha$ altos no son una prueba contundente de que una escala sea unidimensional, es necesario probar su adecuación con otras medidas.

La adecuación de la escala de modos de afrontamiento fue probada mediante los análisis de regresión, donde la variable por predecir fue el estrés y las de predicción, el afrontamiento y la percepción de apoyo social. Los coeficientes que evaluaron la adecuación del modelo fueron muy semejantes en todos los casos, pero la tolerancia indicó colinearidad, que ocurre cuando dos o más medidas correlacionan muy alto, en otras palabras, miden lo mismo de tal manera que puede ser reagrupada en una sola.

En conjunto, los niveles de fiabilidad elevados y el análisis de regresión múltiple mostraron que no es posible sostener que la escala de modos de afrontamiento es multidimensional y por el con- trario, sí es unidimensional. Una prueba más es la evaluación por mitades donde se puede considerar que los errores del azar operan de la misma manera en ambas partes y, por lo tanto, se supone un nivel de ejecución de los encuestados, homogéneo a lo largo de toda la prueba (Cortada, 2000), lo que se confirma por los coeficientes elevados de Guttman y Spearman-Brown.

Desde el punto de vista teórico, la escala fue construida a partir de la definición de afrontamiento, pero la estructura teórica no tiene elementos para ir más allá del afrontamiento dirigido al problema o la emoción; la expectativa que tenían Lazarus y Folkman (1991) de encontrar conceptos nuevos, a partir de la factorización del instrumento, no fue viable. La literatura correspondiente al afrontamiento muestra la tendencia a ser específica del contexto de aplicación, esto es, cuando el afrontamiento es medido de acuerdo con la situación en la que la intervención tiene lugar, (p.e. afrontamiento en el trabajo, la escuela, relación de pareja, entre otros) los resultados mejoran; sin embargo, poca información presentan respecto de las propiedades métricas de estos instrumentos. Estudios más exhaustivos, al respecto, son necesarios.

\section{Referencias}

Aldwin, C. M. \& Revenson, T. A. (1987). Does Coping Help? A Reexamination of the Relation between Coping and Mental Health. Journal of Personality and Social Psychology, 53, 337-348.

Aliaga F. \& Capafóns, A. (1996). La medida del afrontamiento: revisión crítica de la "escala de modos de coping" (Ways of coping). Ansiedad y estrés, 2(1), 17-26.

Atkinson, M. \& Violato, C. (1994). Neuroticism and coping with anger: The trans-situational consistency of coping responses. Personality and Individual Differences, 17(6), 769-782.

Cohen, S., Gottlieb, B. \& Underwood, L. (2000). Theoretical and historical perspectives. En S. Cohen, L. Underwood \& B. Gottlieb (Eds.), Social support measurement and intervention: A guide for health 
and social scientists (pp. 3-28). New York: Oxford University Press.

Cohen, S. \& Wills, T. (1985). Stress, social support, and buffering hypothesis. Psychological Bulletin, 98(2), 310-357.

Cortada, N. (1968). Manual para la construcción de test objetivos de rendimiento. Buenos Aires: Paidós.

Cortada, N. (2000). Técnicas psicológicas de evaluación y exploración. México: Trillas.

Dunkel-Schetter, Ch., Folkman, S. \& Lazarus, R. S. (1987). Correlates and social support receipt. Journal of Personality and Social Psychology, 53, 71-80.

Folkman, S. \& Lazarus, R. S. (1980). An analysis of coping in a middle-aged community sample. Journal and Health and Social Behavior, 21, 219-239.

Folkman, S. \& Lazarus, R. S., (1985). If it changes, it must be a process: A study of emotion and coping during three stages of a college examination. Journal of Personality and Social Psychology, 48, 150-170.

Folkman, S. \& Lazarus, R. S. (1988). Coping as a mediator of emotion. Journal of Personality and Social Psychology, 54, 466-475.

Folkman, S., Lazarus, R. S., Dunkel-Schetter, C., Delongis, A. \& Gruen, R. J. (1986). Dynamics of a stressful encounter: Cognitive appraisal, coping and encounter outcomes. Journal of Personality and Social Psychology, 50(5), 992-1003.

Folkman, S., Lazarus, R. S., Gruen, R. J. \& Delongis, A. (1986). Appraisal, coping, heath status and psychological symptoms. Journal of Personality and Social Psychology, 50(3), 571-579.

Ibáñez, I. \& Olmedo, C. E. (2003). Factorización exploratoria del cuestionario Ways of Coping (WCQ). Psicología conductual. Revista Internacional de Psicología Clínica y de la Salud, 1, 79-94.

Lakey, B. \& Cohen, S. (2000). Social support theory and measurement. En S. Cohen, L. Underwood \& B. Gottlieb (Eds.), Social support measurement and intervention: A guide for health and social scientist (pp. 29-52). New York: Oxford University Press.

Lazarus, R. S. \& Folkman, S. (1984/1991). Estrés y procesos cognitivos. México: Martínez Roca.
Nava, C., Anguiano, S. \& Vega, Z. (2004). Adaptación y fiabilidad del Inventario de estrés cotidiano: un estudio transcultural. Revista Internacional de Psicología Clínica y de la Salud, 12 (2), 323-331.

Nunnally, J. \& Bernstein, I. (1995). Teoría Psicométrica (3ae ed.). México: McGraw- Hill.

Parkes, K. P. (1984). Locus of control, cognitive appraisal, and coping in stressful episodes. Journal of Personality and Social Psychology, 46, 655-668.

Parkes, K. P. (1986). Coping in stressful episodes: the role of individual differences, enviromental factors, and situational characteristics. Journal of Personality and Social Psychology, 51, 1277-1292.

Parker, D. A., Endler, N. S. \& Bagby, R. M. (1993). If it changes, it might be unstable: Examining the factor structure of the Ways of Coping Questionnaire. Psychological Assessment, 5(3), 361-368.

Scheier, M. F., Weintraub, J. K. \& Carver, Ch. S. (1986). Coping with stress: Divergent strategies of optimists and pessimists. Journal of Personality and Social Psychology, 51, 1257-1264.

Sotelo, A. C. \& Maupome, S. V. (1999). Traducción y estandarización del cuestionario modos de afrontamiento al estrés de Lazarus y Folkman, para una población de adolescentes mexicanos. Tesis de Licenciatura UNAM, México D.F., México.

Vaux, A., Phillips, J., Holly, L., Thomson, B., Williams, D. \& Stewart, D. (1986). The social support appraisals (SSA) scale: Studies of reliability and validity. American Journal of Community Psychology, 14, 195-200.

Vitalino, P. P., Russo, J., Carr, J. E., Maiuro, R. D. \& Becker, J. (1985). The ways of coping cheklist: Revision and psychometric properties. Multivariate Behavioral Research, 20, 3-26.

Wills, T. \& Shinar, O. (2000). Measuring perceived and received social support. En S. Cohen, L. Underwood \& B. Gottlieb (Eds.), Social support measurement and intervention: A guide for health and social scientists (pp. 86-135). New York: Oxford University Press. 
